\title{
The mechanism of nanostructuring upon nanosecond laser irradiation of a STM tip
}

\author{
J. Boneberg, H.-J. Münzer, M. Tresp, M. Ochmann, P. Leiderer
}

Universität Konstanz, D-78457 Konstanz, Germany

Received: 20 April 1998/Accepted: 2 June 1998

\begin{abstract}
The mechanism of nanostructuring by illumination of scanning probe tips is examined. For that purpose the tip of a scanning tunneling microscope is illuminated with nanosecond and femtosecond laser pulses. The observation of a transient increase of the tunneling current on the timescale of $\mu s$ is indicative of thermal expansion, which amounts to several $\mathrm{nm}$ for the energy density necessary for the purpose of nanostructuring. Furthermore, quantized electrical resistance can be observed upon illumination, which shows the formation of mechanical contact between tip and surface. Thus it is concluded that the appearance of nanostructures (craters or mounds) is dominated by the cohesion properties of the tip/sample combination. Finally it is shown that even a huge increase of the involved electromagnetic field, reached by the reduction of the pulse length from $10 \mathrm{~ns}$ to $100 \mathrm{fs}$, does not change this scenario.
\end{abstract}

PACS: 42.62.-b; 61.16.Ch; 65.70.+y

Recent experiments have shown the possibility of surface nanostructuring by illumination of a scanning microscope tip with ns laser pulses [1-5]. This seems to be a promising method since a high degree of reproducibility has been reported [3-5]. Furthermore this method is applicable to different materials (for example $\mathrm{Au}, \mathrm{Au} / \mathrm{Pd}$, Ti, PMMA, polycarbonate, organic media) as well as with different kinds of microscopes (AFM and STM) [3-5]. However, the responsible mechanism is controversial. On one hand field enhancement in the vicinity of the tip and subsequent field evaporation is proposed, while thermal effects are ruled out [1-5]. On the other hand mechanical contact as a result of thermal expansion is discussed as well [6-8]. Such thermal effects have also been observed in other combinations of pulsed laser light with scanning microscope tips [9-11].

We performed transient measurements of the tunneling current during such experiments, which should enable us to distinguish between these two models due to the different time scales involved. Whereas a field evaporation mechanism should take place on the time scale of the laser pulse (ns), a thermal effect occurs with a typical cooling time in the order of $\mu$ s (from $\tau=\mathrm{d}^{2} / 2 D$ one can estimate a relaxation time of $73 \mu$ s for a tungsten tip with a thermal diffusivity $D$ of $0.68 \mathrm{~cm}^{2} / \mathrm{s}$ and a focus diameter $\left.d=100 \mu \mathrm{m}\right)$. Following the thermal model it might also be possible to observe indications of the mechanical contact, for example quantized conductance of the junction formed upon illumination. Therefore we present measurements of the gap resistance of a STM upon illumination with a ns laser pulse. Finally a comparison of current transients after illumination with ns and fs laser pulses, respectively, is shown.

\section{Experimental}

All experiments were performed under ambient conditions with a home-built STM using etched PtIr or W tips on thin gold films on mica or glass substrates. For the first part of the measurements a mildly focused Q-switched Nd:YAG laser pulse was applied for the illumination $(0.1 \mathrm{~mm}$ diameter $)$. The angle of incidence was $75^{\circ}$ with respect to the tip axis. The frequency-doubled pulse $(532 \mathrm{~nm})$ with a pulse width of $7 \mathrm{~ns}$ (full width at half maximum) was used. The energy density of the pulse was controlled by two thin film polarizers, in order to keep the beam profile constant.

For the comparison of ns/fs laser pulses a Ti:sapphire laser system was used. This system consists of a frequencydoubled cw Nd:YAG laser, a Ti:sapphire oscillator and a regenerative amplifier, pumped by a Q-switched frequencydoubled Nd:YAG. The laser system emits 100-fs pulses of energies up to $10 \mathrm{~mJ}$ at a wavelength of $800 \mathrm{~nm}$. The regenerative amplifier can be used without the injection of a seed pulse and emits then 10-ns pulses. Therefore a comparison of fs and ns pulses at an identical wavelength is possible. Once again the adjustment of the pulse energy was performed by two thin film polarizers.

The transient current was measured directly with a current amplifier (1 M $\Omega$ resistor in series with the gap resistance and a following voltage amplification of 100) that has a response time on the order of $30 \mu \mathrm{s}$. The gap voltage measurement was performed on the basis of an instrumentation amplifier (BurrBrown INA 110). 


\section{Results and discussion}

For a comparison of our results with other nanostructuring experiments we first determined the threshold energy for the appearance of nanostructures. For this purpose the tip was approached to the surface at typical tunneling conditions of $1 \mathrm{nA}$ and at an applied voltage of $300 \mathrm{mV}$. Figure 1 shows the appearance of a Au surface after the illumination of a $\mathrm{Pt} / \mathrm{Ir}$ tip with a pulse of the energy density $400 \mathrm{~mJ} / \mathrm{cm}^{2}$. A hillock can be observed with a diameter of about $40 \mathrm{~nm}$ and a height of $30 \mathrm{~nm}$. This surface structure as well as the energy density used are similar to earlier studies of other groups [1-5].

Next we measured the transient tunneling current at energy densities in the order of $1 \mathrm{~mJ} / \mathrm{cm}^{2}$, which is distinctly below the threshold for nanostructuring. The result is depicted in Fig. 2 for a tip of $\mathrm{W}$ and PtIr, respectively. At $t=0$ the laser pulse hits the surface (at $t=-1 \mathrm{~ms}$ the feedback loop was switched off) and a steep increase of the tunneling current appears. After reaching a maximum of roughly $5 \mathrm{nA}$ the current decreases once again on a timescale of $280 \mu$ s for the PtIr tip and $60 \mu$ s for the $\mathrm{W}$ tip. The exact value of this relaxation time varies from tip to tip, but nevertheless all values observed in our experiments were above the time resolution of the amplifier (30 $\mu \mathrm{s})$. Similar values for tungsten tips have also been observed by Lyubinetsky et al. [7].

Two main points have to be considered here. First, the observed timescale, in particular for the PtIr tips, is above the laser pulse length. This is already a strong hint that this effect is not due to a field effect but is due to thermal expansion. Second, the timescale observed depends on the tip material.

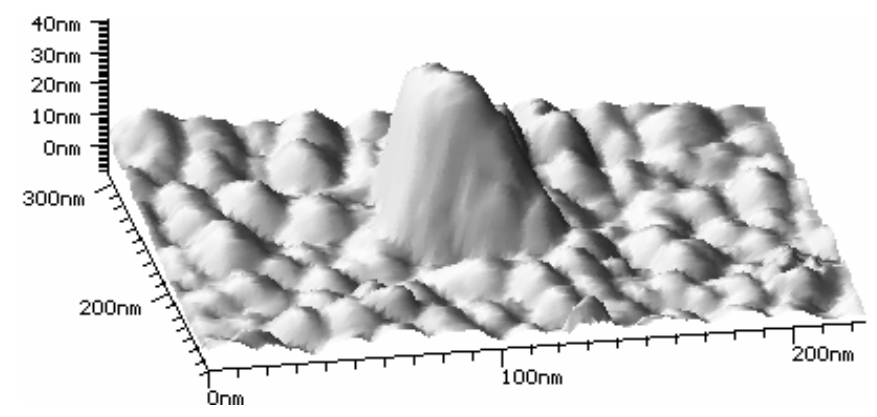

Fig. 1. Surface topography of a Au film after the illumination of a Pt/Ir tip with a laser pulse of the energy density $400 \mathrm{~mJ} / \mathrm{cm}^{2}$

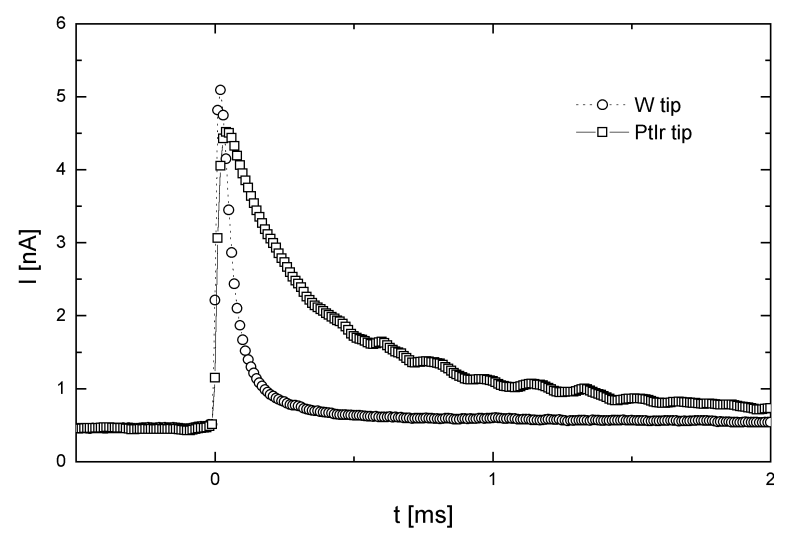

Fig. 2. Transient tunneling current after illumination of a PtIr and a W tip with a laser pulse of the energy density $1 \mathrm{~mJ} / \mathrm{cm}^{2}$
Since the applied energy density was only slightly different for the two different tip materials, this implies that the main contribution is due to thermal expansion of the tip. We attribute the difference in the relaxation times to the different thermal parameters of the two materials. From the absolute values of the current changes we can make a crude estimation of the expansion involved. A current increase of one order of magnitude corresponds to a thermal expansion of several Ångstroms under ambient conditions already at energy densities of $1 \mathrm{~mJ} / \mathrm{cm}^{2}$. From a more detailed analysis of the transient current [8] one can determine that at the threshold for nanostructuring the expansion amounts to several $\mathrm{nm}$. Thus it can be concluded that mechanical contact is established.

The occurrence of mechanical contact should lead to quantization effects in the conductivity and thus in the gap voltage, which we want to focus on in the following. The behaviour of the gap voltage is depicted in Fig. 3 for increasing energy densities of the laser pulse. The gap voltage reveals a pronounced minimum on a time scale of $100 \mu$ s. For energy densities above $10 \mathrm{~mJ} / \mathrm{cm}^{2}$ the voltage drops to zero within our accuracy. A further increase of the energy density leads to the formation of a plateau. The width of this plateau increases with pulse energy. Then the gap voltage increases back to the applied bias voltage, while the $I-V$ converter is still in saturation at this time. A similar behaviour for tunneling current and gap voltage was found for tungsten tips.

It has to be noted that the energy densities used in this experiment are still well below the threshold for the appearance of nanostructures. Nevertheless the disappearance of the gap voltage indicates that an electrical, and hence also a mechanical, contact between tip and surface is established. Consequently it might be possible to measure quantized resistance during the formation and disconnection of the contact. In the thermal model the expansion has a maximum at the end of the laser pulse. The approach towards the surface happens on the timescale of the laser pulse (10 ns) and cannot be resolved by the temporal resolution of our experimental setup. The experiment indicates, however, that the retraction occurs on a much longer time scale. Therefore we studied the increase of the gap voltage after the plateau in detail. Figure 4 a shows an expanded view of the time-resolved resistivity in this region. Some traces for the same energy density of $200 \mathrm{~mJ} / \mathrm{cm}^{2}$

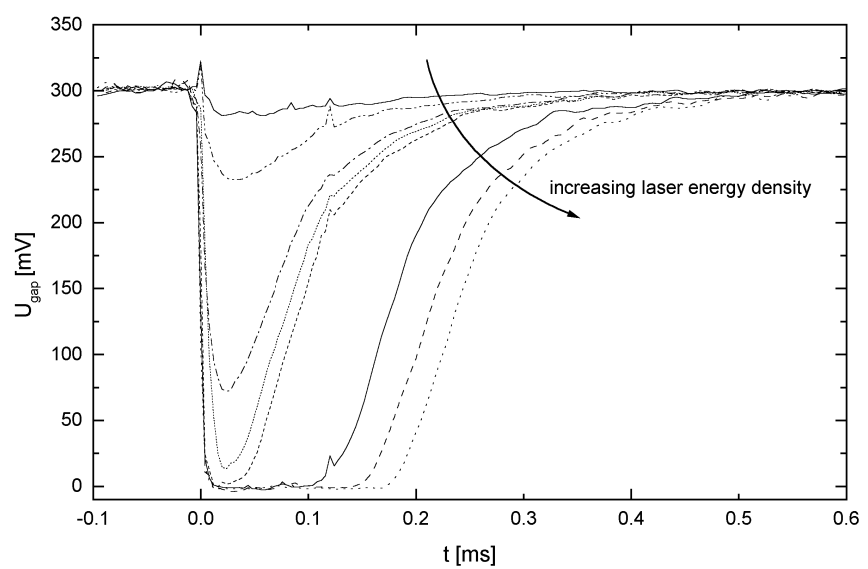

Fig. 3. Transient behaviour of the gap voltage after illumination of a $\mathrm{Pt} / \mathrm{Ir}$ tip with the energy densities $5,6,7.6,8.6,10,10.6,11.6,12.6 \mathrm{~mJ} / \mathrm{cm}^{2}$ 
are given [12] (for a better visibility the different traces are horizontally shifted against one another). Several steps can be found in these traces. A correlation with multiples of $2 e^{2} / h$ (marked by horizontal lines) is obvious, as is also shown in the conductivity histogram (Fig. 4b). It should be mentioned that the traces are not typical for a series of several shots. In general the steps are clearly visible in one out of five.

A comparison with calculations from the literature allows to conclude that a contact was formed. The observation of multiples of $2 e^{2} / h$ is expected during the breaking of the contact [13-16]. Despite this contact formation we could not detect the formation of nanostructures below energy densities of $400 \mathrm{~mJ} / \mathrm{cm}^{2}$.

The experiment was then repeated with tungsten tips. Although the time-resolved gap voltage characteristics exhibited a similar behaviour as in Fig. 3 , no steps at $2 e^{2} / h$ could be observed during the experiments. It is remarkable that, together with the absence of the quantized resistance, the appearance of the nanostructures formed at higher energy densities by $\mathrm{W}$ tips is also changed compared to the PtIr tips. Instead of hillocks, formed during a laser illumination process with PtIr, holes are observed upon laser illumination of the W tip.

This appearance of mounds and craters is also well known from nanostructuring experiments where contact was established [17] or voltage pulses were applied $[18,19]$. In some experiments it was already concluded that field emission is not the crucial process for the pattern formation [18-20]. Alternatively it was suggested that the extent of cohesion between tip and surface and a subsequent plastic deformation is

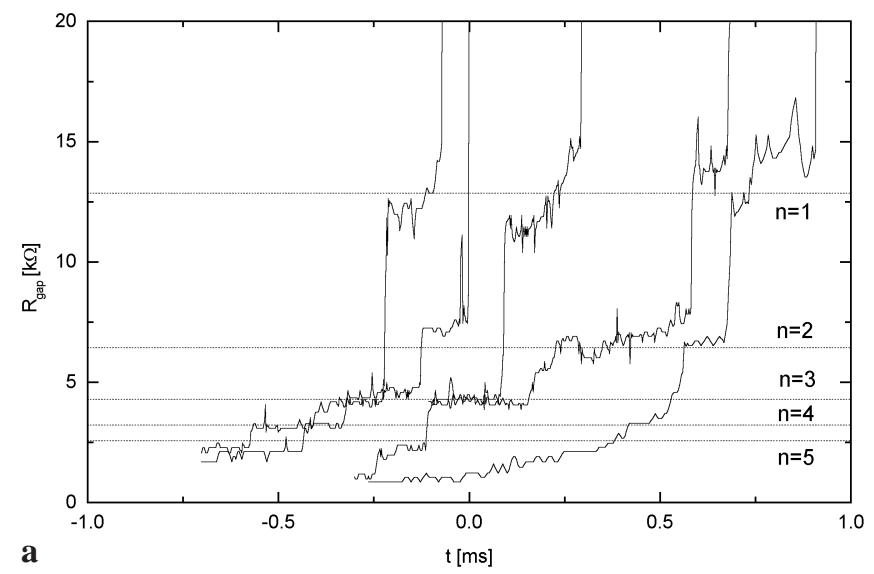

$\mathbf{a}$

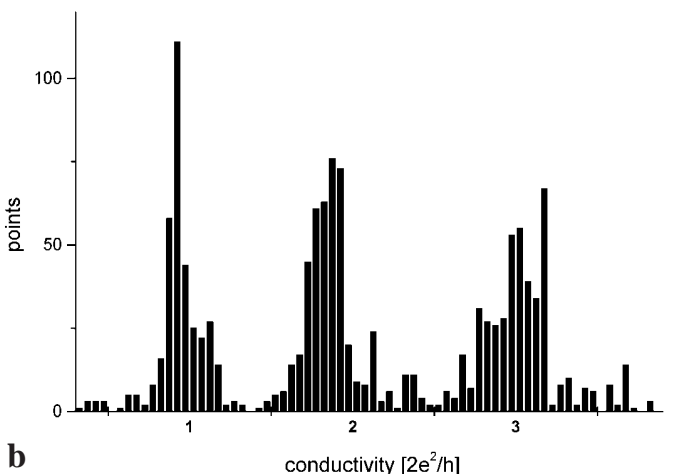

Fig. 4. a Time-resolved resistivity during the breaking of the contact. b Histogram of the resistivity values observed in a

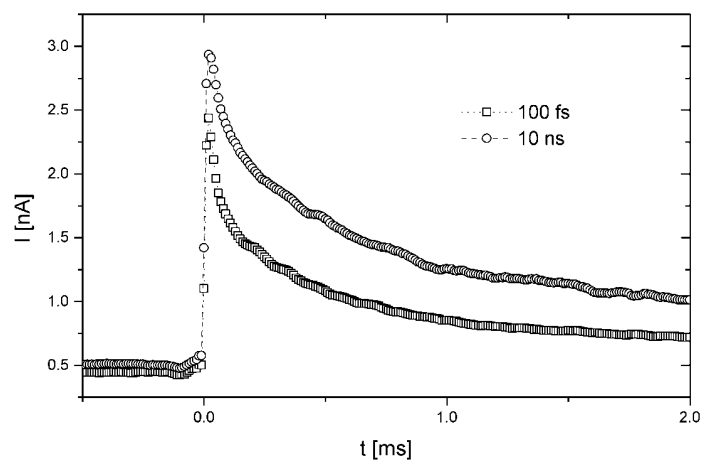

Fig. 5. Transient tunneling current after illumination of a PtIr tip with 10-ns and 100-fs laser pulses

the crucial parameter of the pattern formation [17, 19]. This suggestion agrees completely with the observations made in our experiments. In the case of high cohesion forces a neck is formed between tip and surface. During the retraction this neck is elongated and quantized resistance is observed. After the breaking of the contact a hillock remains at the surface. On the other hand for low cohesion forces no neck is formed and thus no quantization effects can be observed. The resulting structure is a crater. Besides this difference in the formed structure, the appearance of quantization without the creation of nanostructures indicates the importance of a further parameter in the nanostructuring mechanism, which has not been discussed up to now. This may be the transition from elastic to plastic deformation, but a final conclusion cannot be drawn from our measurements at the present time.

Up to this point we could not find any hint on fieldinduced effects, although calculations predict pronounced field enhancement below the tip [21-23]. Obviously the response of the tip under ns illumination is completely dominated by thermal effects. A possible approach to reduced thermal effects at the same electromagnetic field intensities is the use of shorter laser pulses. Therefore we compared the transient currents upon illumination with 10 -ns versus $100-$ fs pulses (Fig. 5). The traces show an almost identical transient behaviour. Once again these measurements were performed at energy densities well below the threshold for nanostructuring. Nevertheless thermal effects are dominating the response of the tip.

\section{Summary}

We believe that the observations presented here complete the model for the formation of nanostructures upon pulse laser illumination of tips in the vicinity of surfaces. Thermal expansion leads to an approach of tip and surface. Contact is formed already at energy densities well below the threshold for the formation of nanostructures. Depending on the cohesion conditions of the tip/surface combination quantized conductance can be observed during the retraction. At higher energy densities quantized conductance appears and mounds are formed during the retraction in the case of large cohesion forces, whereas craters are formed in the opposite case. Therefore the creation of nanostructures seems to be a pure thermal/mechanical effect. Although the field enhancement below the tip may be a large effect for certain tip materials, it 
is completely covered by the fast thermal expansion involved at the energy densities used. Field effects may becomes accessible in the case of large tip-sample distances or with fs pulses and tip materials of small thermal expansion.

Acknowledgements. This work was supported by the Deutsche Forschungsgemeinschaft (SFB 513).

\section{References}

1. A.A. Gorbunov, W. Pompe: Phys. Status Solidi A 145, 333 (1994)

2. K. Dickmann, J. Jersch: Laser Optoelektron. 27, 76 (1995)

3. J. Jersch, K. Dickmann: Appl. Phys. Lett. 68, 868 (1996)

4. J. Jersch, F. Demming, K. Dickmann: Appl. Phys. A 64, 29 (1997)

5. J. Jersch, F. Demmig, L.J. Hildenhagen, K. Dickmann: Appl. Phys. A 66, 29 (1998)

6. V.A. Ukraintsev, J.T. Yates, Jr.: J. Appl. Phys. 80, 2561 (1996)

7. I. Lyubinetsky, Z. Dohnálek, V.A. Ukraintsev, J.T. Yates, Jr.: J. Appl. Phys. 82, 4115 (1997)

8. J. Boneberg, M. Tresp, M. Ochmann, H.-J. Münzer, P. Leiderer: Appl. Phys. A 66, 615 (1998)
9. R. Huber, M. Koch, M. Perner, W. Spirkl, J. Feldmann: Proceedings of the Conference on Lasers and Electro-Optics 1998, Technical Digest, CFH4, San Francisco, CA, May 1997

10. V. Gerstner, A. Knoll, J. Lehmann, M. Merschdorf, W. Pfeiffer, A. Thon, G. Gerber: Verhandl. DPG (VI) 33, O34.48 (1998)

11. Ch. Lienau, A. Richter, T. Elsaesser: Appl. Phys. Lett. 96, 325 (1996)

12. A slightly higher energy density was used here in order to achieve a pronounced effect on the timescale of the resolution of our measurement

13. J.I. Pascual, J. Méndez, J. Gómez-Herrero, A.M. Baró, N. Garcia, U. Landmann, W.D. Luedtke, E.N. Bogachek, H.-P. Cheng: Science 267, 1795 (1995)

14. J.M. Krans, J.M. van Rultenbeek, V.V. Flans, I.K. Yanson, L.J. de Jongh: Nature 375, 767 (1995)

15. J.A. Torres, J.J. Senz: Phys. Rev. Lett. 77, 2245 (1996)

16. I.P. Batra: Surf. Sci. 395, 43 (1998)

17. J.K. Gimzewski, R. Möller: Phys. Rev. B 36, 1284 (1987)

18. C.X. Guo, D.J. Thomson: Ultramicroscopy 42-44, 1452 (1992)

19. T.C. Chang, C.S. Chang, H.N. Lin, T.T. Tsong: Appl. Phys. Lett. 67, 903 (1995)

20. J.I. Pascual, J. Méndez, J. Gómez-Herrero, A.M. Baró, N. Garcia: Phys. Rev. Lett. 71, 1852 (1993)

21. W. Denk, D.W. Pohl: J. Vac. Sci. Technol. B 9, 510 (1991)

22. O.J.F. Martin, C. Girard: Appl. Phys. Lett. 70, 705 (1997)

23. L. Nowotny, R.X. Bian, X.S. Xie: Phys. Rev. Lett. 79, 645 (1997) 